\title{
Neonatal Rupture of the Spleen: Successful Treatment with Splenic Artery Embolization
}

\author{
Jelle W. Raats, MD, $\mathrm{PhD}^{1}$ Lievay van Dam, $\mathrm{MD}^{2}$ Pieter J. van Doormaal, $\mathrm{MD}^{2}$ \\ Marjoleine van Hengel-Jacobs, MD, PhD $^{3}$ Hester Langeveld-Benders, MD PhD ${ }^{1}$
}

${ }^{1}$ Department of Pediatric Surgery, Erasmus University Medical Center, Sophia Children's Hospital, Rotterdam, Netherlands

${ }^{2}$ Department of Interventional Radiology, Erasmus University Medical Center, Rotterdam, Netherlands

3 Department of Pediatrics, Division of Neonatology, Erasmus University Medical Center, Sophia Children's Hospital, Rotterdam, Netherlands
Address for correspondence Jelle Raats, MD, PhD, Department of Pediatric Surgery, Erasmus University Medical Center, Sophia Children's Hospital, Doctor Molewaterplein 40, Rotterdam, The Netherlands (e-mail: jelle.raats@gmail.com).

Am J Perinatol Rep 2021;11:e58-e60.

\begin{abstract} Neonatal intra-abdominal hemorrhage has been rarely reported in the literature. We Keywords

- splenic injury

- endovascular treatment

- interventional radiology

- neonatal care

- intra-abdominal hemorrhage

- surgical emergency report a case of splenic injury in a neonate, highlighting the importance of a high-index suspicion in early recognition of this rare and potentially fatal injury. We report the first case of a neonate who had a splenic rupture and underwent successful endovascular treatment.
\end{abstract}

\section{Case Report}

The patient was born after a 38-week twin gestation and weighing $2,875 \mathrm{~g}$. . He was born second via vaginal breech delivery. The Apgar's scores were 6 of 10 at 1 minute and 8 of 10 at 5 minutes. He briefly received positive end-expiratory pressure because of suspected respiratory distress syndrome and was transferred to the maternity ward the same day. Later that day, he developed feeding difficulties, looked pale, and became less reactive. The serum hemoglobin concentration was $8.1 \mathrm{mmol} / \mathrm{L}$. As bleeding or early onset of sepsis was suspected, resuscitation was started with $10 \mathrm{mg} / \mathrm{kg}$ of $0.9 \%$ normal saline and antibiotics. Additional laboratory testing ruled out hemolysis and showed a further decrease in hemoglobin concentration to $4.5 \mathrm{mmol} / \mathrm{L}$. Ultrasound was performed in search for a bleeding source and confirmed the presence of a hemoperitoneum ( - Fig. 1). After having received a double red blood cell transfusion $(7.5 \mathrm{mg} / \mathrm{kg})$ and $1 \mathrm{mg}$ of fytomenadion intravenously, the baby was transferred to the neonatal intensive care unit (NICU) of the Erasmus MC-Sophia Children's Hospital, Rotterdam, the Netherlands.

On arrival at the NICU, he was hemodynamically stable and without respiratory support. Although he looked pale, he was comfortable, awake, and alert. His abdomen was tender with muscular defense but he was neither distended nor discolored. Nonoperative management was attempted with further correction of coagulopathy. Overnight, the baby received

July 4, 2020

accepted after revision

January 28, 2021
DOI https://doi.org/

$10.1055 / \mathrm{s}-0041-1727257$

ISSN 2157-6998.

\footnotetext{
(C) 2021. The Author(s).

This is an open access article published by Thieme under the terms of the Creative Commons Attribution-NonDerivative-NonCommercial-License, permitting copying and reproduction so long as the original work is given appropriate credit. Contents may not be used for commercial purposes, or adapted, remixed, transformed or built upon. (https://creativecommons.org/ licenses/by-nc-nd/4.0/)

Thieme Medical Publishers, Inc., 333 Seventh Avenue, 18th Floor, New York, NY 10001, USA
} 


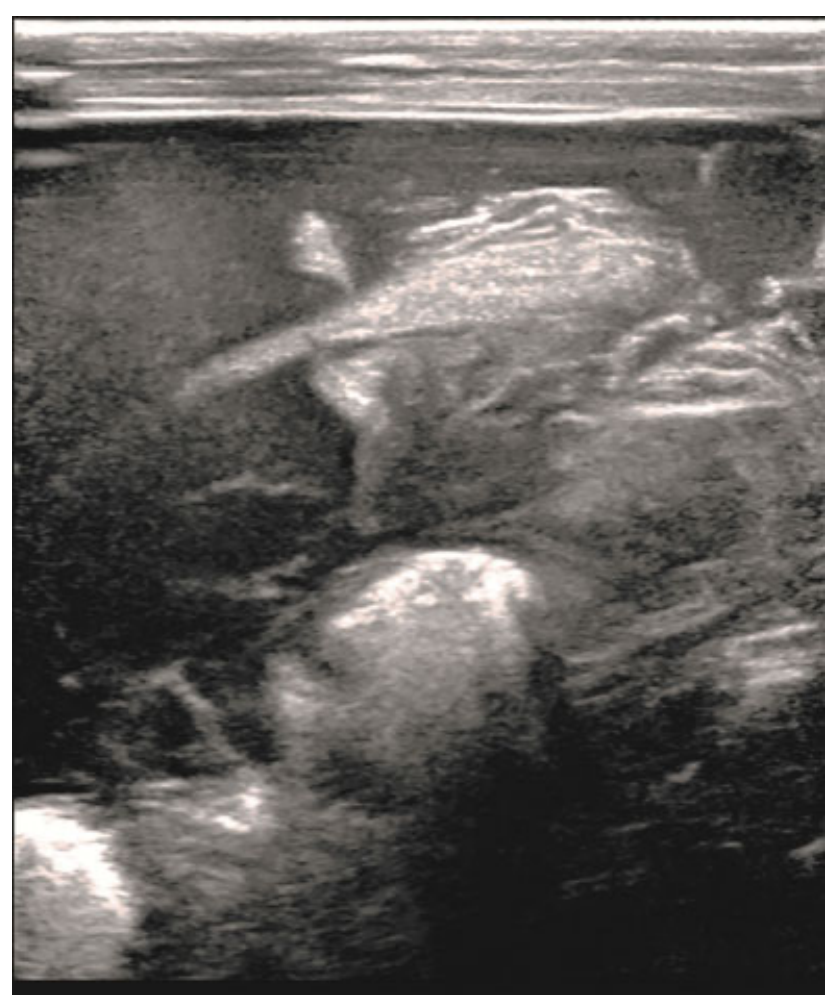

Fig. 1 Ultrasound confirmed the presence of a hemoperitoneum.

deteriorated and was given more blood product. Increasing abdominal distension and abdominal tenderness was noted, and the baby hardly had any urine production. Bedside ultrasound confirmed the presence of a hematoperitoneum with normal appearance of the liver and retroperitoneum including both adrenals. In addition, a large heterogenic hematoma was observed in the splenic fossa with distortion of the craniodorsal part of the spleen (-Fig. 2), suggestive or a large splenic rupture. During the beside ultrasound study, the baby deteriorated further and needed urgent intubation. Ventilation became difficult because of the mass effect of the hematoperitoneum. We opted for coiling of the splenic artery to treat the bleeding instead of laparotomy. We had argued that the bleeding might worsen if we would release the abdominal tension by performing an emergency laparotomy without treating the bleeding first, and that the baby could die from exsanguination. He was transferred to the interventional radiology department for angiography and endovascular treatment of the splenic bleeding.

Using a broadband $12-\mathrm{MHz}$ linear array transducer (L12-5; Philips Medical, Eindhoven, the Netherlands), the common femoral artery was measured $1.8 \mathrm{~mm}$. Under ultrasound guidance, the common femoral artery was punctured with a 21-gauge needle, and a 4-French (Fr) radial artery sheath (Merit Medical, Salt Lake City, UT) was introduced. Using a 4-Fr cobra-shaped catheter (C2 Dadiofocus Glidecath Terumo, Tustin, CA), the celiac trunk was catheterized. An angiography of the celiac trunk was performed, and this showed vasospasm of the splenic artery. No active contrast extravasation was observed. The splenic artery was catheterized with a 1.7-Fr microcatheter (Excelsior SL-10, Stryker,

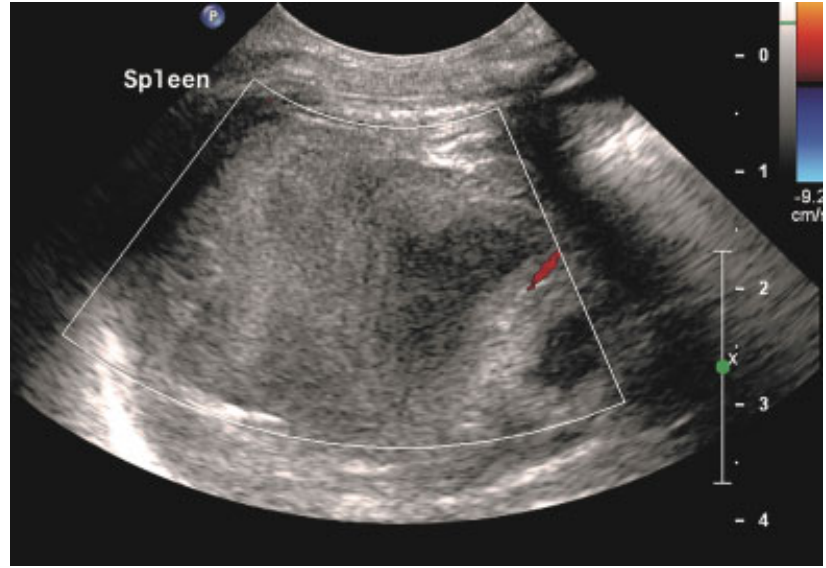

Fig. 2 Ultrasound showed a heterogenic hematoma with distortion of the craniodorsal part of the spleen.

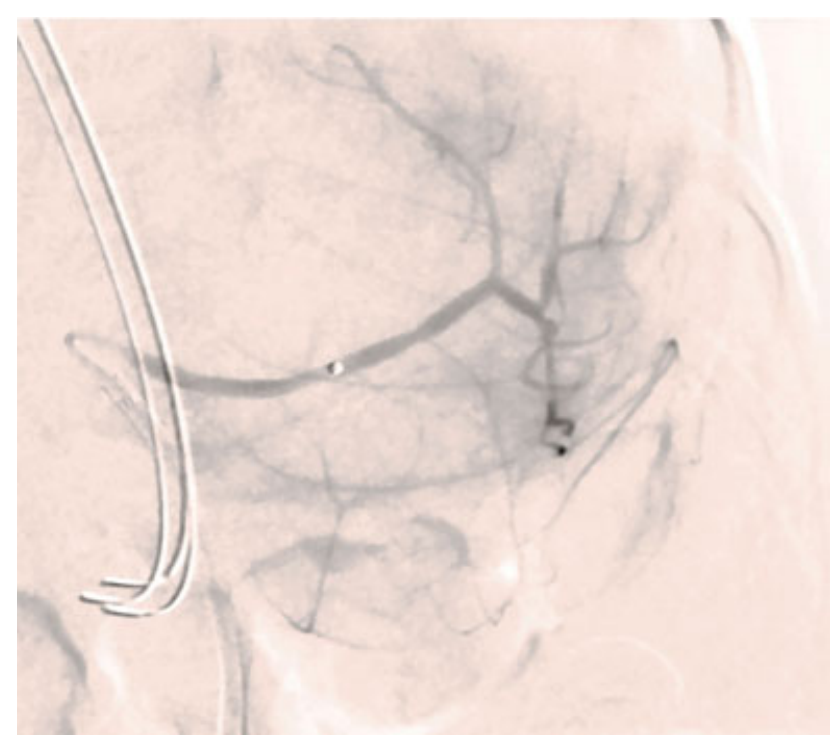

Fig. 3 A selective angiogram of the splenic artery showed spasm of both the splenic artery and the intra-splenic vessels.

Fremont, CA) and 0.014" hydrophilic guidewire (Traxcess, Terumo, Tustin, CA). A selective angiogram of the splenic artery showed spasm of both the splenic artery and the intrasplenic vessels. No focal bleeding was observed (-Fig. 3). After multidisciplinary consultation, we decided to embolize the proximal splenic artery using multiple $1 / 1 \mathrm{~mm}$ detachable NanoCoils (Stryker) to relieve the pressure of the splenic vascular bed, and thereby to preserve future splenic function. Postembolization angiography showed an adequate occlusion of the proximal splenic artery, with collateral flow to the distal splenic artery through the short gastric arteries (-Fig. 4).

To treat the distended abdomen caused by the hematoperitoneum, resulting in ventilation difficulties and potential risk of an abdominal compartment syndrome, a 6.3-Fr pigtail drain (Cook Medical, Bloomington, IN) was placed in the right lower abdominal quadrant and a total of 320-cc blood was drained during the first 24 hours. 


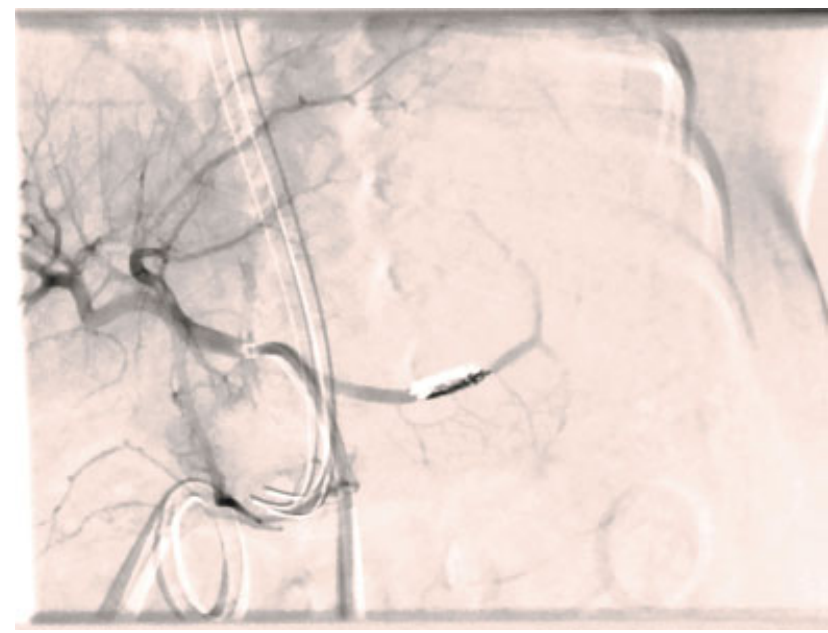

Fig. 4 Postembolization angiography showed an adequate occlusion of the proximal splenic artery.

After the intervention, the boy became hemodynamically stable and ventilation problems were dissolved.

The boy was discharged home 10 days after the intervention. An abdominal ultrasound performed 2 weeks later showed a small hematoma around the spleen. The spleen had a normal appearance of the parenchyma on the ultrasound and had a length of $31 \mathrm{~mm}$.

\section{Discussion}

Neonatal intra-abdominal hemorrhage has been rarely reported in the literature. Diagnosing intra-abdominal bleeding in a newborn is difficult, and diagnosis is often made at autopsy. ${ }^{1}$ The triad of pallor, anemia, and abdominal distension are mostly described in literature. ${ }^{1}$ The presentation of intra-abdominal bleeding can be variable with nonspecific symptoms such as feeding difficulties, pallor, tachycardia, polypnea, or scrotal hematoma. ${ }^{2}$

Some of the differentials entertained in this case included hemorrhagic disease of the newborn, sepsis with disseminated intravascular coagulation, as well as solid organ injury. The latter included adrenal injury, liver injury, as well as a splenic injury. Splenic injury is the least common of these conditions. The pathophysiology of splenic injury during related to delivery is not fully understood. Hemoperitoneum secondary to splenic rupture is most often due to stretching of the splenorenal ligament and is usually associated with dystocia and difficulties of extraction. ${ }^{3}$ The mechanism involved could be an elevated fetal intrathoracic pressure during uterine contractions, which pushes the liver and spleen out of the diaphragmatic cavity, resulting in an excessive strain to their supporting ligaments. ${ }^{4}$ In this case, the mother had experienced the delivery as traumatic.
However, no instruments to assist the delivery had been used. It is highly likely that the difficulties during extraction had caused the splenic injury.

Some authors suggest performing exploratory laparotomy as soon as possible when the diagnosis is confirmed in the newborn.

Splenic rupture in a neonate is a rare surgical emergency associated with high mortality. ${ }^{1}$ When an emergency laparotomy is performed, in most cases, a splenectomy is required to control the bleeding. A splenectomy, however, carries the risk of an overwhelming sepsis. In this case, endovascular treatment was performed by proximal embolization of the splenic artery. As reported in the systematic review of Rong et al, this is related to less life-threatening complications than distal embolization of the splenic artery. ${ }^{5}$

\section{Conclusion}

We report the first case of a neonate who had a splenic rupture and underwent a successful endovascular treatment. In this case of our patient, endovascular treatment was performed, despite the fact that invasive extensive surgery was most probably unavoidable. Endovascular treatment with splenic artery embolization of neonatal rupture of the spleen should always be considered when expertise and resources are available.

Financial Disclosure

All authors have no financial relationships relevant to this article to disclose.

\section{Funding Source}

No funding was secured for this study.

Conflict of Interest

All authors have no conflicts of interest to disclose.

\section{References}

1 Ting JY, Lam BC, Ngai CS, Leung WC, Chan KL. Splenic rupture in a premature neonate. Hong Kong Med J 2006;12(01):68-70

2 Tiboni S, Abdulmajid U, Pooboni S, Wighton C, Eradi B, Dagash H. Spontaneous splenic hemorrhage in the newborn. European J Pediatr Surg Rep 2015;3(02):71-73

3 Descamps CS, Cneude F, Hays S, et al. Early hypovolemic shock and abdominal distention due to neonatal splenic rupture: urgency of diagnosis and management. Eur J Pediatr 2017;176(09): $1245-1250$

4 Hui CM, Tsui KY. Splenic rupture in a newborn. J Pediatr Surg 2002;37(04):E3

5 Rong JJ, Liu D, Liang M, et al. The impacts of different embolization techniques on splenic artery embolization for blunt splenic injury: a systematic review and meta-analysis. Mil Med Res 2017; $4: 17$ 